\title{
High-energy pulsars, from gamma rays to gravitational waves
}

\section{Massimiliano Razzano*}

Dipartimento di Fisica "E. Fermi", Universita' di Pisa, and INFN-Pisa, Italy

E-mail: massimiliano.razzanodpi.infn.it

\section{Giovanna Pivato}

Dipartimento di Fisica "E. Fermi", Universita' di Pisa, and INFN-Pisa, Italy

E-mail: giovanna.pivatoepi.infn.it

\section{On behalf of the Fermi-LAT Collaboration}

In its first six years of operations, the Fermi Gamma-ray Space Telescope has revolutionized our view of the gamma-ray Universe. Thanks to its unprecedented performance, the Large Area Telescope onboard Fermi has detected more than two thousand sources and unveiled new classes of gamma-ray emitters. In particular, pulsars are the main contributors to the gamma-ray source population in our Galaxy and are relevant not only to astrophysics. In fact, they are excellent natural laboratories to probe the laws of physics under extreme conditions, e.g. of gravity and electromagnetic fields. Among the population of the new gamma-ray pulsars detected by Fermi, the young, energetic ones are also very promising candidate sources of continuous gravitational waves. I will review the recent Fermi results on pulsars and discuss the possible perspectives for multimessenger observations of these sources

Science with the New Generation of High Energy Gamma-ray experiments, 10th Workshop 04-06 June 2014

Lisbon - Portugal

\footnotetext{
*Speaker.
} 


\section{Introduction}

Since its launch in June 2008, the Fermi Large Area Telescope (LAT) has dramatically changed our view of $\gamma$-ray pulsars. The LAT is a pair-conversion telescope consisting of a silicon tracker, an electromagnetic calorimeter and a segmented anticoincidence detector [ $[$ ] . It detects $\gamma$ rays from $20 \mathrm{MeV}$ to more than $300 \mathrm{GeV}$, covering also the energy range from $10 \mathrm{GeV}$ to $100 \mathrm{GeV}$, that was largely unexplored by previous $\gamma$-ray telescopes. The main improvements with respect to its predecessor, the EGRET experiment onboard the Compton Gamma Ray Observatory, are a large effective area ( $\sim 8000 \mathrm{~cm}^{2}$ at $1 \mathrm{GeV}$ on-axis), a sharp Point Spread Function (PSF, $\mathrm{R}_{68 \%} \sim 0.6^{\circ}$ at $1 \mathrm{GeV}$ on-axis), a large field of view $(\sim 2.4 \mathrm{sr})$, and excellent absolute timing $(<1 \mu \mathrm{s})$. These features lead to an improvement in sensitivity of a factor of $\sim 20$ with respect to EGRET. The Fermi observatory is in a low-altitude orbit with a period of $\sim 95$ minutes: this orbit, combined with the survey-based observing strategy, guarantees complete coverage of the full sky every 2 orbits, i.e. $\sim 3$ hours. Among the $\gamma$-ray sources detected by the LAT, pulsars are the most numerous in our Galaxy. In about 6 years of observations, $\sim 160 \gamma$-ray pulsars have been detected, 117 of them included in the Second Fermi LAT catalog of $\gamma$-ray pulsars[四][2PC]. These sources are almost evenly divided in three subgroups: 1) young, radio-loud pulsars, 2) young, radio-quiet objects, and 3) radio-loud millisecond pulsars. $\gamma$ rays are powerful probes to understand the physics of the pulsar machine. First of all, the $\gamma$-ray domain is where the bulk of the electromagnetic radiation is emitted: in comparison, only a tiny fraction is emitted as radio waves. Furthermore, $\gamma$ rays are emitted along magnetic field lines with a small pitch angle, allowing for a precise tracking of the geometry of the magnetosphere. Also, the $\gamma$-ray emission beam differs from the radio one. This means that $\gamma$ rays can be used to map different populations of radio-loud and radio-quiet pulsars in the Galaxy. Finally, neutron stars are the endpoint of stellar evolution, and the study of these remnants of massive stars is closely linked to that of the pulsar wind nebula and supernova remnant cosmic-ray accelerators with which they are sometimes co-located. With the continuous detection of more $\gamma$-ray pulsars, the LAT is thus contributing to a better understanding of the pulsar contribution to the stellar population of the Galaxy.

\section{Pulsar searches with Fermi LAT}

Due to its less precise angular resolution compared to X-ray, optical and radio telescopes, it is not easy to identify pulsars only using spatial coincidence. For this reason, alternative approaches had to be developed to successfully find $\gamma$-ray pulsars. Currently, three main ways are available: 1) for pulsars already known at other wavelengths (e.g. radio), phase folding the $\gamma$ rays to check if they are modulated at the same rotation frequencies as seen in radio ,2) blind searches and 3) blind searches over a limited parameter space, exploiting the existing information from observations at other wavelengths (e.g. optical or X-rays).

Blind searches 2) and 3) are particularly challenging because $\gamma$ rays are extremely sparse and the LAT detects a pulsed photon every millions of rotational periods for the weakest radio pulsars detected. The search over long datasets makes the Fourier-based techniques impractical because of the large amount of CPU time needed. To reduce this problem, the time-differencing technique was developed [ []]. It is based on computing the Fast Fourier Transform of the differences between the 
$\gamma$-ray arrival times in a sliding window whose width can be as large as the entire data epoch. The blind searches are even more challenging for binary pulsars, the case for many Millisecond Pulsars (MSPs). A full search over the 6 Keplerian parameters is extremely challenging and unfeasible for the current computing facilities. But even in the case of isolated MSPs, positional uncertainties become more relevant with increasing rotation frequency: the typical tolerance, using several years of data, is of the order of an arc second. These reasons show why blind searches for MSPs are particularly challenging.

To overcome these problems, observations at other wavelengths are particularly valuable. For instance, X-ray observations can be used to pinpoint a point-like counterpart and reduce the uncertainties related to the position. Moreover, optical observations of the stellar companion of MSPs can help to constrain the orbital parameters of the binary system, thus reducing the phase space for the search. This approach has been successful for the discovery of PSR J1311-3430 [15]]. This source is an example of "black widow", i.e. an eclipsing binary MSPs with very compact, circular orbit where the pulsar wind is ablating its low-mass stellar companion [ए8]].

\section{Models for $\gamma$-ray emission}

One of the main objectives of the study of pulsars is to understand what is the mechanism that explains $\gamma$-ray emission. Although high-energy radiation indicates the presence of high energy charged particles, it is not clear where the particles get accelerated. One possibility is to consider vacuum gaps, where the electric fields of the magnetospheric plasma is not screened, and particles can therefore be accelerated.

Three classes of models emerged according to the location of the emission region. The Polar Cap (PC) models [Q] assume that charge acceleration takes place in the polar cap, and the radiation comes from low altitudes above the polar caps of the neutron star. The emitted $\gamma$ rays can be absorbed in the high-intensity magnetic fields above the poles, creating a sharp, super-exponential cutoff in the energy spectrum.

The second group of emission models require that the emitting region comes from the outer magnetosphere, in particular in the Outer Gaps (OG) or in the Slot Gap/Two Pole Caustic (SG/TPC) models. The OG model [ए7] posits outer-magnetospheric gaps reaching from the null charge surface (i.e. $\Omega \cdot B=0$ ). According to the SG/TPC models [四], particles can be accelerated and emit radiation from the surface up to the light cylinder, i.e. where the co-rotation speed equals the speed of light. Some constraints on these models can be made by looking at the phase-averaged spectrum, in order to understand the emission characteristics. The LAT is sensitive enough to $\mathrm{GeV}$ energies to measure the shape of the energy cutoff and distinguish between PC and outer-magnetospheric models. In Figure $\mathbb{W b}$ the spectral energy distribution of the Vela pulsar (PSR J0835-4510) is shown: the PC model predicts a sharp turnover at $\sim 10 \mathrm{GeV}$ due to attenuation of the $\gamma$-ray flux in the magnetic field. Figure Wa shows that the spectral change, that happens at $\sim 2.9 \mathrm{GeV}$, is not well reproduce by the PC model, that has to be rejected [వ].

\section{The population of $\gamma$-ray pulsars}

Figure $\$$ shows the state of art of the known $\gamma$-ray pulsar population in the second catalog of 

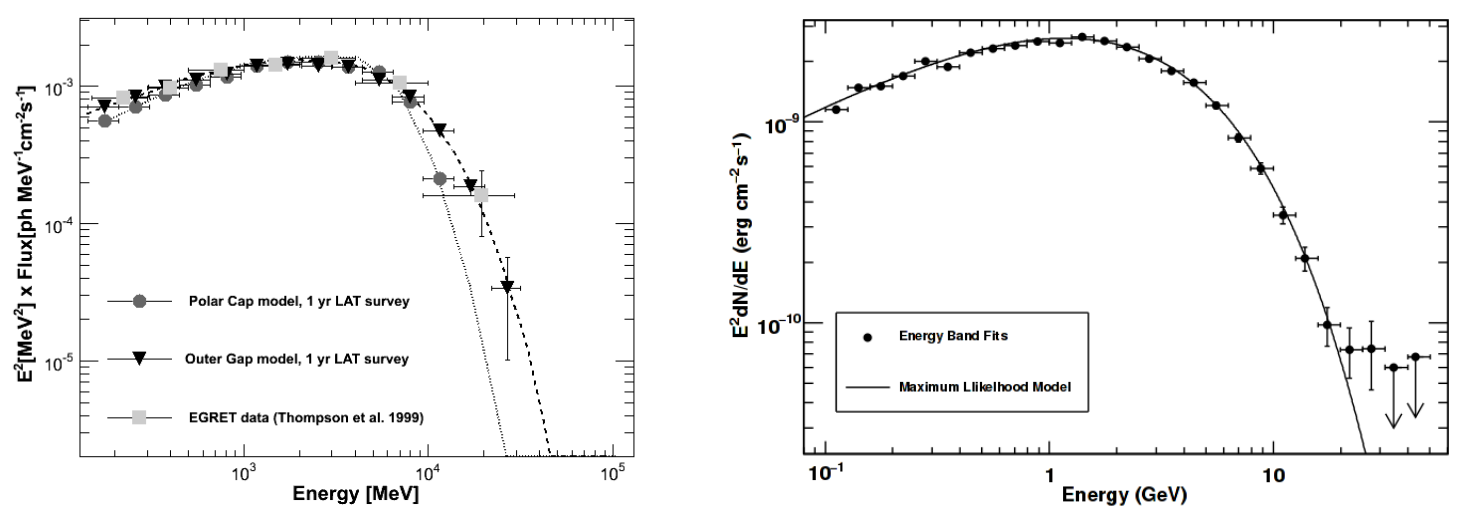

Figure 1: a) Vela spectrum as observed by EGRET, superimposed with the simulation of two different models (Razzano et al. 2009): the polar cap and the outer magnetosphere. b)Vela spectrum from []].

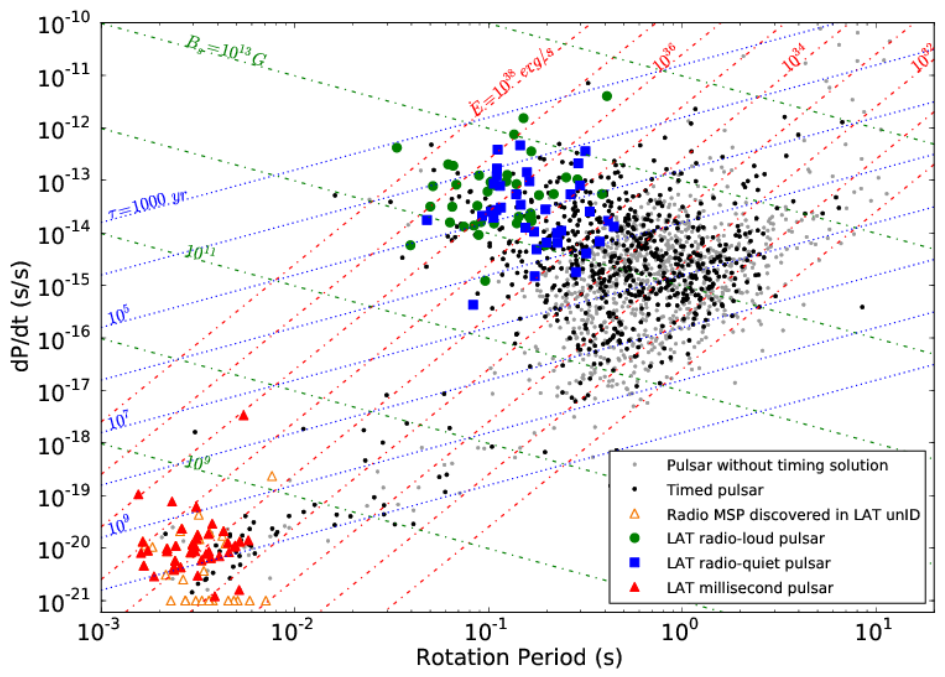

Figure 2: $P-\dot{P}$ distribution of the Fermi pulsars plotted with the entire radio pulsar sample known today. Lines indicate constant rotational energy loss $\dot{E}$, surface B field, and characteristic age. From [四].

Fermi pulsars (2PC). The plot shows a clear separation between radio-quiet pulsars, dominating the range from $10^{33}$ to $10^{35} \mathrm{erg} \mathrm{s}^{-1}$, and radio-loud pulsars, with spindown power $\dot{E}>10^{37} \mathrm{erg} \mathrm{s}^{-1}$. The presence of a separate, older, population of Millisecond Pulsars (MSPs) is evident from the plot in the lower-left corner.

\subsection{Light curves and phase-resolved spectra}

The study of light curves is crucial to determine the characteristics of a pulsar: $\sim 70 \%$ of the young pulsars and $\sim 60 \%$ of the MSPs have two peaks (a leading peak P1 and a trailing P2) and that the ratio $\mathrm{P} 2 / \mathrm{P} 1$ of the peak amplitudes increase with energy, indicating an harder second peak [四]. Young pulsars and MSPs have a P1 $\gamma$-ray peak that lags with respect to the radio counterpart: this lag is greater for MSPs than for isolated neutron stars, indicating that a smaller magnetosphere 
implies a stronger aberration of the radio pulses. Other peculiar characteristics of MSPs are the $\gamma$-ray peaks aligned with radio peaks or leading it. According to the caustic interpretation of the peaks, photons come from different altitudes and arrive at the observer at the same time due to aberration and other relativistic effects.

Furthermore, phase-resolved spectra are of great interest for pulsars and help to sample a number of different local conditions such as magnetic field and curvature radius.

\subsection{Pulsar glitches}

Glitches, i.e. sudden increases of the rotational frequency, are a still debated and not wellunderstood phenomenon in pulsars. A possible explanation of these events is the transfer of angular momentum between a solid crust and a more rapidly rotating component present in the inner part of the neutron star [[2]. In order to study these phenomena, the monitoring capability of Fermi to catch glitches on the fly and to characterize all the phases is of crucial importance.

An example of the signature of a glitch is shown in figure 3: in this plot, the phase changing is evident in the changing of position of the peak in the phase-time plot.

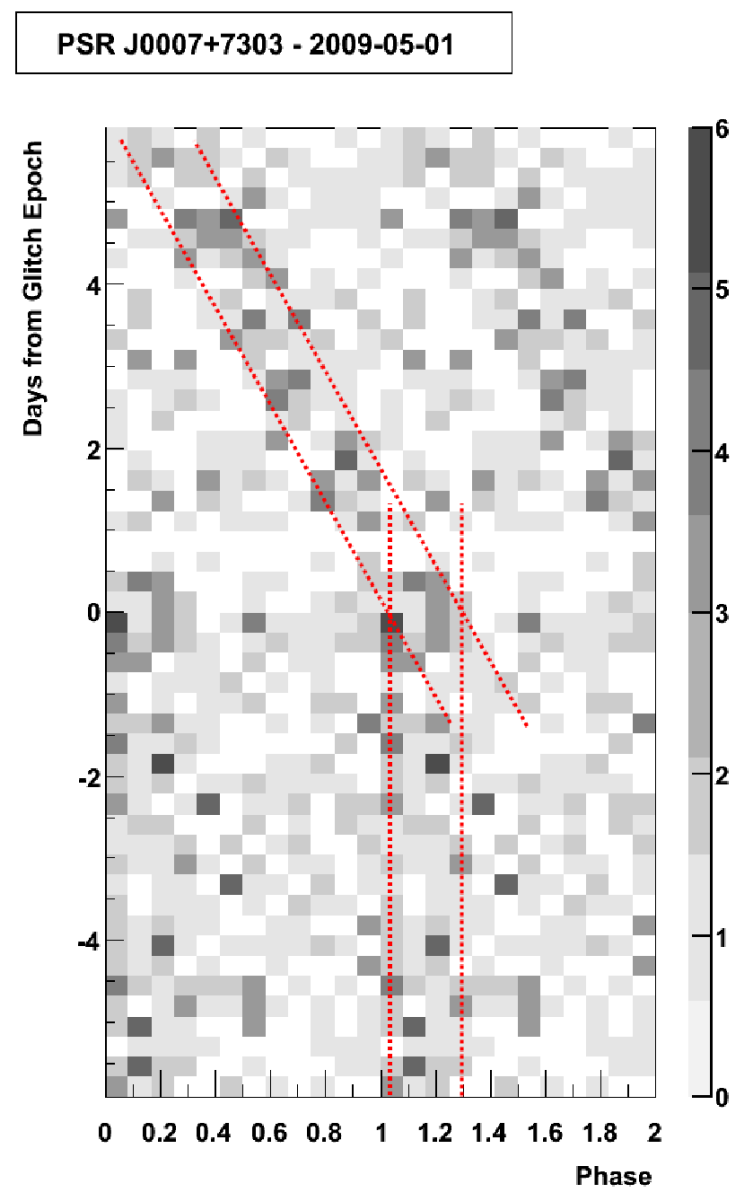

Figure 3: Phase-time diagram of the pulsar J0007+7303 showing the typical signature of a glitch. From [12] 


\subsection{Pulsar variability}

It has been taken as an axiom that pulsars are steady in $\gamma$ rays on timescales longer than the longer than the days or weeks that it takes for the LAT to detect a signal. However, Fermi LAT unveiled two pulsars showing mode changes, namely PSR J2021+4026 in the Gamma Cygni Supernova Remnant and PSR J1023+0038, considered the "missing link" between pulsars and Xray binaries. PSR J2021+4026 showed a decrease in flux in less than a week, simultaneous with an increase in the spin-down rate. During this event, a change in light curve and spectrum has been observed. Mode changes and intermittent behavior are known for radio pulsars, but the one of PSR $\mathrm{J} 2021+4026$ is of crucial importance because it is the first in $\gamma$ rays.

The origin of this behavior is still debated but a possible explanation is that the change in pulsed

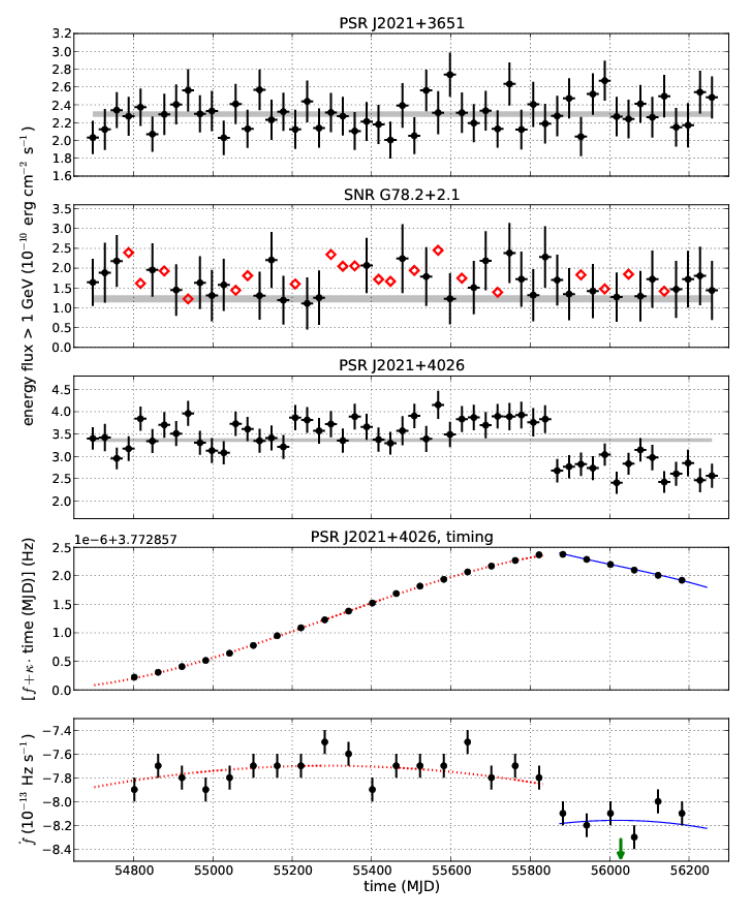

Figure 4: a) From top panel down: energy flux $(\mathrm{E}>1 \mathrm{GeV}$ ) divided into 30 day bins for J2021+3651, SNR G78.2+2.1, and J2021+4026. The first two sources are shown to prove the non-contamination of Gamma Cygni dataset: the variation in flux is only visible for $\mathbf{J} 2021+4026$, so it is not an artifact due to contamination of other objects. The gray bands show the average source fluxes for all data. Bottom two panels: for $\mathbf{J} 2021+4026, f+k \cdot$ time (MJD) is reported, with frequency $f$ and $k=6.9^{-8} \mathrm{~Hz}_{\text {day }}{ }^{-1}$, and frequency derivative $\dot{f}$, vs. time, from the periodicity search in 60 day windows.From [B]]. From [प⿴囗十)].

$\gamma$-ray emission is due to a change in geometry or charge currents in the magnetosphere that led to a shift of magnetic field inclination or effective current [B]].

\section{Pulsars and gravitational waves: a multimessenger approach}

The existence of gravitational waves (GWs) was confirmed in an indirect way in 1974 by the observations of PSR B1913+16 made by Russel Hulse and Joseph Taylor. This object is a binary 
system composed of a 59-ms pulsar and a neutron star, and its orbit is 7.7 hours. Observations shows that the evolution of the period of the orbits is in agreement with the energy loss due to the emission of gravitational waves. However, this binary pulsar provides an indirect way of detecting gravitational waves (GWs). A direct detection is still to come, but it may become reality in the near future thanks to the second-generation interferometers Virgo and the Laser Interferometer Gravitational Observatory (LIGO). Virgo is located in Cascina, near Pisa, and has 3-km long, while LIGO consists of two twin, 4-km long, Interferometers located in Hanford, WA and Livingston, LA. The most promising sources of gravitational waves are mergers of compact objects, like neutron stars and/or black holes. However, continuous, periodic gravitational waves are expected from asymmetric pulsars. Pulsars are thus a perfect target for multi-messenger observations. In fact, gravitational radiation teaches us a lot about the distribution of the mass of the neutron star, while the electromagnetic observations are crucial to investigate the physics of pulsar magnetospheres. In this multimessenger context many Fermi pulsars are young and energetic so they are good candidates to detect GWs. In addition Fermi has a continuous monitor of the sky, providing timing solution of pulsars, the radio-quiet ones. Further information, in the lower frequencies, are provided by the timing arrays. According to some theoretical models, also pulsar glitches are potential candidate sources of gravitational waves, although the signal may be too small to be detected.

\section{Conclusions}

Pulsars are among the most powerful and eclectic objects in the Universe, and they are natural laboratories to probe fundamental physics and are of crucial importance to understand emission processes. They are the major contributors to the $\gamma$-ray population of the Galaxy and their diversity can offer a viable way to constrain models of neutron star magnetosphere. They are important not only for a better knowledge of the physics mechanism for the production of the emission, but also they are possible targets for advanced gravitational interferometers in the coming era of gravitational astronomy.

\section{Acknowledgements}

The Fermi LAT Collaboration acknowledges support for LAT development, operation and data analysis from NASA and DOE (United States), CEA/Irfu and IN2P3/CNRS (France), ASI and INFN (Italy), MEXT, KEK, and JAXA (Japan), and the K.A. Wallenberg Foundation, the Swedish Research Council and the National Space Board (Sweden). Science analysis support in the operations phase from INAF (Italy) and CNES (France) is also gratefully acknowledged.

\section{References}

[1] Abdo et al., ApJS, 208, 17, 17 (2003)

[2] Abdo et al., ApJ, 696, 1084 (2009)

[3] Allafort et al., ApJL, 777, L2 (2013)

[4] Arons, ApJ, 266, 215 (1993) 
[5] Atwood et al., ApJL, 652, 1071 (2006)

[6] Atwood et al, ApJ, 32, 913 (2009)

[7] Caraveo, Annual Review, 52, 211 (2014)

[8] Cheng et al., ApJ, 300, 500 (1986)

[9] Daugherty et al., ApJ, 458, 278 (1996)

[10] Deutsch, Ann. d'Astrophys., 18, 1 (1955)

[11] Goldreich et al., ApJ, 157, 869 (1969)

[12] Link et al., Nature, 359, 616 (1992)

[13] Muslimov et al., ApJ, 588, 430 (2003)

[14] Muslimov et al., ApJ, 606, 1143 (2004)

[15] Pletsch, H. J., Guillemot, L., Fehrmann, H., et al. 2012, Science, 338, 1314

[16] Razzano, M., Harding, A. K., Baldini, L., et al. 2009, Astroparticle Physics, 32, 1

[17] Romani, ApJ, 470, 469 (1996)

[18] Saz Parkinson et al., Proceeding IAU Symposium, 291 (2012)

[19] Stappers et al., The Astronomer's Telegram, oct, 5513 (2013)

[20] Stopnitzky et al., ApJ, 787, 114 (2014)

[21] Spitkovsky, ApJ, 648, L51 (2006)

[22] Venter et al., Astronomische Nachrichten, 335, 268 (2014) 